\title{
Escola, família e o diabetes tipo 1: sentidos produzidos pelas Mães Pâncreas
}

\author{
School, family and type 1 diabetes: meanings produced by pancreatic mothers \\ Escuela, familia y diabetes tipo 1: significados producidos por las madres páncreas
}

Recebido: 21/01/2022 | Revisado: 26/01/2022 | Aceito: 29/01/2022 | Publicado: 31/01/2022

\author{
Cláudia Marcele de Campos \\ ORCID: https://orcid.org/0000-0003-0779-4846 \\ Universidade de Cuiabá, Brasil \\ E-mail: claudia.campos@ifmt.edu.br \\ Lucy Ferreira Azevedo \\ ORCID: https://orcid.org/0000-0002-7072-3859 \\ Universidade de Cuiabá, Brasil \\ E-mail: lucyfazevedo@gmail.com
}

\begin{abstract}
Resumo
Este trabalho constitui-se como um recorte da pesquisa de dissertação do Mestrado, em andamento, vinculado ao Programa de Pós-graduação Stricto Sensu em Ensino UNIC-IFMT, cujo tema central são os sentidos produzidos pelos atores envolvidos no processo ensino-aprendizagem de crianças com diabetes mellitus tipo 1, estudantes do ensino fundamental de escolas públicas de Cuiabá-MT. O diabetes é uma doença crônica cuja incidência ocorre predominantemente na infância e na adolescência, que aumenta a cada ano, mas que ainda é desconhecida pela escola brasileira. O objetivo deste artigo é refletir sobre os sentidos produzidos pelas Mães Pâncreas, as principais cuidadoras das crianças com DM1, sobre a relação escola-família e seus efeitos na aprendizagem desses estudantes. A metodologia adotada para este estudo é de abordagem qualitativa, de cunho interacionista sociodiscursivo, por meio da investigação da produção de sentidos enunciados pelas práticas discursivas dos participantes. Os resultados parciais demonstram que as instituições escolares não têm oferecido a assistência necessária ao pleno desenvolvimento dos estudantes com DM1, sobrecarregando as Mães Pâncreas física e emocionalmente. Diante do exposto, espera-se, com este estudo, levantar a discussão sobre a importância da elaboração de políticas públicas voltadas a estudantes com condições especiais de saúde, mas que não se encaixam no público-alvo atendido pela educação especial.
\end{abstract}

Palavras-chave: Ensino; Diabetes; Produção de sentidos.

\begin{abstract}
This article is an excerpt from the Master's dissertation research, in progress, linked to the Stricto Sensu Postgraduate Program in Teaching UNIC-IFMT, whose central theme is the meanings produced by the actors involved in the teaching-learning process of children. with type 1 diabetes mellitus, elementary school students from public schools in Cuiabá-MT. Diabetes is a chronic disease whose incidence occurs predominantly in childhood and youth, which increases every year, but which is still unknown by Brazilian schools. The purpose of this article is to reflect on the meanings produced by Pancreatic Mothers, the main caregivers of children with DM1, on the school-family relation and its effects on these students' learning. The methodology adopted for this study is of a qualitative approach, of a socio-discursive interactionist nature, through the investigation of the production of meanings enunciated by the discursive practices of the participants. The partial results show that school institutions have not offered the necessary assistance for the full development of students with DM1, overloading the Pancreatic Mothers physically and emotionally. It is expected, with this study, to raise the discussion about the importance of developing public policies aimed at students with special health conditions, but who do not fit into the target audience served by special education.
\end{abstract}

Keywords: Teaching; Diabetes mellitus; Production of senses.

\section{Resumen}

Este artículo es un extracto de la investigación de disertación de Maestría, en curso, vinculada al Programa de Posgrado en Enseñanza Stricto Sensu UNIC-IFMT, cuyo tema central son los significados producidos por los actores involucrados en el proceso de enseñanza-aprendizaje de los niños con diabetes tipo 1, estudiantes de enseñanza básica de escuelas públicas de Cuiabá-MT. La diabetes es una enfermedad crónica cuya incidencia ocurre predominantemente en la niñez y la adolescencia, que aumenta cada año, pero que aún es desconocida por las escuelas brasileñas. El propósito de este artículo es reflexionar sobre los significados producidos por las Madres Páncreas, principales cuidadoras de niños con DM1, sobre la relación escuela-familia y sus efectos en el aprendizaje de estos estudiantes. La metodología adoptada para este artículo es de abordaje cualitativo, de carácter interaccionista sociodiscursivo, por medio de la investigación de la producción de sentidos enunciados por las prácticas discursivas de los participantes. Los resultados parciales muestran que las instituciones escolares no han ofrecido la asistencia 
necesaria para el pleno desarrollo de los estudiantes con DM1, sobrecargando física y emocionalmente las Madres Páncreas. Se espera, con este estudio, suscitar la discusión sobre la importancia de desarrollar políticas públicas dirigidas a estudiantes con condiciones especiales de salud, pero que no encajan en el público objetivo atendido por la educación especial.

Palabras clave: Enseñanza; Diabetes mellitus; Producción de sentidos.

\section{Introdução}

O diabetes mellitus, ou simplesmente diabetes, ${ }^{1}$ é uma doença crônica grave que acomete cerca de 537 milhões de pessoas ao redor do mundo. Dentre as quatro categorias de classificação ${ }^{2}$ existentes para essa patologia, o diabetes mellitus tipo 1 (DM1), é o de maior incidência entre crianças e adolescentes, embora possa acometer pessoas de qualquer idade.

De acordo com a décima edição do Atlas do Diabetes, publicado pela Federação Internacional de Diabetes (2021), o DM1 corresponde a cerca de $10 \%$ do total dos casos de diabetes em todo o mundo, número que cresce ano após ano. Atualmente, considerando-se a faixa etária de 0 a 19 anos, o Brasil é o $3^{0}$ país do mundo tanto em número de incidência (novos casos) como de prevalência (casos já existentes), ficando atrás apenas da Índia e dos Estados Unidos. Em números absolutos, nosso país possui mais de 92 mil crianças e adolescentes vivendo com diabetes.

Apesar de não ser uma doença rara, ela é pouco conhecida pela maior parte da população brasileira. De maneira geral, as pessoas sabem muito pouco sobre diabetes, e o que pensam que sabem nem sempre está correto. Não é exagero afirmar que até mesmo pessoas bem próximas ao DM1 (como amigos e alguns familiares) reproduzem informações desatualizadas, equivocadas e sem comprovação científica alguma. Dito em outras palavras, muitos ainda acreditam em "mitos". Para exemplificar, a maioria das pessoas desconhece que o DM1 é uma doença autoimune, cuja manifestação não é possível evitar, e associam sua incidência ao consumo exacerbado de açúcar, o que está completamente equivocado.

O tratamento do DM1 é complexo, caro e pode ser exaustivo. A rotina de cuidados exige o domínio de uma série de conhecimentos, além da observação de muitas variáveis. Além da aplicação de insulina, o tratamento envolve controle da alimentação, prática de atividade física e monitoramento da glicemia (SBD, 2020). Tudo isso é bastante complexo para uma criança, por isso acaba sendo assumido pelas mães, que popularmente se autodenominam Mães Pâncreas, já que é o papel dessa glândula que elas tentam reproduzir com a aplicação de insulina várias vezes ao dia.

Conforme exposto anteriormente, a pessoa com diabetes possui uma condição de saúde séria e incurável, mas que não impõe limitações à sua aprendizagem. Assim, a criança diabética, como toda criança e adolescente brasileiro, também possui direito à educação visando ao pleno desenvolvimento de suas potencialidades (Brasil, 1990; 1996). E isso não se resume a ter acesso aos conteúdos do currículo escolar. À criança DM1 também deve ser garantido o direito de frequentar e de se apropriar de todos os espaços escolares.

Além disso, a escola é fundamental para o desenvolvimento moral e social da criança, para a compreensão do senso de coletividade, do respeito à diversidade, da aprendizagem de valores essenciais para a vida em sociedade (Vinha, 2016). A pandemia deixou nítida a importância da escola para a formação cidadã e, por tudo isso, nenhuma criança deve ser privada de frequentá-la, independentemente de suas particularidades.

Diante desse contexto, o objetivo deste estudo é apresentar os sentidos produzidos pelas mães sobre a relação escolafamília de crianças diagnosticadas com DM1, estudantes do ensino fundamental de escolas públicas de Cuiabá-MT.

\footnotetext{
${ }^{1}$ Substantivo que aceita dois gêneros e dois números, ou seja, são igualmente aceitos os vocábulos o diabete, a diabete, o diabetes e a diabetes. Neste trabalho, adotaremos o termo o diabetes.

2 Pode-se classificar o diabetes em quatro categorias: Diabetes Mellitus tipo 1 (DM1), Diabetes Mellitus tipo 2 (DM2), Diabetes Gestacional e Outros Tipos. (Maraschin, Murussi, Witter \& Silveiro, 2010). Neste estudo, ênfase dar-se-á sobre o DM1.
} 


\section{Aspectos Metodológicos do Estudo Realizado}

Este trabalho constitui-se como um recorte da pesquisa de dissertação do Mestrado, em andamento, vinculado ao Programa de Pós-graduação Stricto Sensu em Ensino UNIC-IFMT, cujo tema central são os sentidos produzidos pelos atores envolvidos no processo ensino-aprendizagem de crianças com DM1, estudantes do ensino fundamental de escolas públicas de Cuiabá-MT.

A metodologia adotada para este estudo é de abordagem qualitativa, de cunho interacionista sociodiscursivo, por meio da investigação da produção de sentidos enunciados pelas práticas discursivas dos participantes.

Guerra (2014) destaca que, na abordagem qualitativa, o objetivo do pesquisador é compreender os fenômenos que estuda, seja ações individuais, grupos ou organizações em seu contexto social, interpretando-os segundo suas próprias perspectivas, sem se importar com valores numéricos, estatísticas e relações lineares de causa e efeito.

Outro conceito importante para este estudo é o de sentido. De acordo com Spink (2010, p. 34),

O sentido é uma construção social, um empreendimento coletivo mais precisamente interativo, por meio do qual as pessoas, na dinâmica das relações sociais, historicamente datadas e culturalmente localizadas, constroem os termos a partir dos quais compreendem e lidam com as situações e fenômenos a sua volta.

Para ampliar a compreensão sobre os resultados, é fundamental entender a concepção de práticas discursivas norteadora desta investigação. Segundo Spink (2010, p. 27), práticas discursivas corresponde "às maneiras pelas quais as pessoas, por meio da linguagem, produzem sentidos e posicionam-se em relações sociais cotidianas". A autora complementa: “implicam ações, seleções, escolhas, linguagens, contextos, enfim, uma variedade de produções sociais das quais são expressão. Constituem, dessa forma, um caminho privilegiado para entender a produção de sentido no cotidiano.” (Spink, 2013, p. 20-21).

Os resultados serão apresentados por meio do diálogo entre o aporte teórico da pesquisa e os sentidos produzidos pelos participantes.

\subsection{Participantes}

Neste recorte, a ênfase será sobre os dados coletados junto às mães, que fazem parte do grupo de participantes da pesquisa de Mestrado em andamento anteriormente mencionada. Foram selecionadas por terem preenchido os critérios de terem um filho diagnosticado com diabetes mellitus tipo 1 que fosse estudante do ensino fundamental de alguma escola municipal localizada na cidade de Cuiabá-MT. Todas participaram voluntariamente da pesquisa.

O perfil socioeconômico das participantes será apresentado no Quadro 1 a seguir. Para preservar a identidade delas, serão identificadas neste estudo como Mãe Pâncreas 1, 2, 3 e 4.

Quadro 1 - Perfil socioeconômico das participantes.

\begin{tabular}{|l|c|c|l|l|c|c|c|}
\hline Identificação & Idade & $\begin{array}{l}\text { Idade do } \\
\text { filho } \\
\text { DM1 }\end{array}$ & Escolaridade & Profissão & $\begin{array}{c}\text { Renda familiar } \\
\text { (salários mínimos) }\end{array}$ & $\begin{array}{l}\text { Custeio do } \\
\text { tratamento }\end{array}$ & $\begin{array}{c}\text { Assistência médica } \\
\text { regular } \\
\text { (criança DM1) }\end{array}$ \\
\hline Mãe Pâncreas 1 & 40 anos & 7 anos & $\begin{array}{l}\text { Ensino } \\
\text { superior } \\
\text { completo }\end{array}$ & Autônoma & 4 a 6 & $\begin{array}{l}\text { SUS + } \\
\text { família }\end{array}$ & Sim \\
\hline Mãe Pâncreas 2 & 32 anos & 6 anos & $\begin{array}{l}\text { Ensino } \\
\text { superior } \\
\text { completo }\end{array}$ & Do lar & 4 a 6 & $\begin{array}{l}\text { SUS + } \\
\text { família }\end{array}$ & Sim \\
\hline Mãe Pâncreas 3 & 47 anos & 9 anos & $\begin{array}{l}\text { Ensino médio } \\
\text { completo }\end{array}$ & Autônoma & 1 a 3 & Família & Não \\
\hline Mãe Pâncreas 4 & 50 anos & 12 anos & $\begin{array}{l}\text { Ensino médio } \\
\text { completo }\end{array}$ & Do lar & 1 & SUS & Sim \\
\hline
\end{tabular}

Fonte: Autores (2022). 
Embora sucinto, o quadro acima traz informações relevantes sobre o acesso da criança DM1 a insumos e acompanhamento médico em seu tratamento, que terão forte influência nos sentidos produzidos pelas mães, como será apresentado no item 3.2 deste trabalho.

\subsection{Coleta de dados}

Os dados foram coletados durante o segundo semestre de 2021, através da aplicação de questionários contendo questões abertas e fechadas, enviados às participantes por meio eletrônico.

Marconi e Lakatos (2017) afirmam que o questionário possui vantagens como: ampla área de abrangência, alcance de maior número de participantes simultaneamente, economia de tempo e recursos, liberdade para o participante responder no horário que considerar mais oportuno, dentre outras. Porém, também possui algumas desvantagens, tais como: o participante deixar muitas perguntas sem respostas, demorar para devolver ou não devolver o questionário; impossibilidade de auxiliar o participante na compreensão de alguma pergunta; não ser aplicável a pessoas analfabetas, entre outras.

É importante destacar que essa foi a opção escolhida em respeito às recomendações de distanciamento social devido ao cenário de insegurança sanitária provocado pela pandemia da Covid-19.

\section{Resultados e Discussão}

Antes de iniciar a discussão, cuja luz será sobre as práticas discursivas enunciadas pelas Mães Pâncreas, convém contextualizar resumidamente a educação especial no Brasil.

\subsection{Diabetes na escola: o que é ser especial na escola brasileira?}

Milhares de estudantes brasileiros convivem com alguma doença crônica e, consequentemente, com as dificuldades impostas por essa condição especial de saúde. Entretanto, muitos desses estudantes não se encaixam no perfil atendido pela educação especial.

Observando-se a legislação brasileira, tem-se na Lei n 9.394/1996, a Lei de Diretrizes e Bases da Educação (LDB), que especial é a "modalidade de educação escolar oferecida preferencialmente na rede regular de ensino, para educandos com deficiência, transtornos globais do desenvolvimento e altas habilidades ou superdotação." (Brasil, 1996). Por sua vez, a Lei $n^{\circ}$ 13.146/2015, Lei Brasileira de Inclusão da Pessoa com Deficiência, define como "pessoa com deficiência aquela que tem impedimento de longo prazo de natureza física, mental, intelectual ou sensorial, o qual, em interação com uma ou mais barreiras, pode obstruir sua participação plena e efetiva na sociedade em igualdade de condições com as demais pessoas". (Brasil, 2015). Além desses dispositivos, outro bastante importante é o Decreto $\mathrm{n}^{\circ} 10.502$, de 30/09/2020, que instituiu a Política Nacional de Educação Especial: Equitativa, Inclusiva e com Aprendizado ao Longo da Vida - PNEE 2020 e estabeleceu as novas diretrizes nacionais para a educação especial.

O Art. $6^{\circ}$ da PNEE 2020 indica qual é o seu público-alvo:

I - educandos com deficiência, conforme definido pela Lei no 13.146, de 6 de julho de 2015 - Estatuto da Pessoa com Deficiência; II - educandos com transtornos globais do desenvolvimento, incluídos os educados com transtorno do espectro autista, conforme definido pela Lei $\mathrm{n}^{\circ} 12.764$, de 27 de dezembro de 2012; e III - educandos com altas habilidades ou superdotação que apresentem desenvolvimento ou potencial elevado em qualquer área de domínio, isolada ou combinada, criatividade e envolvimento com as atividades escolares. (Brasil, 2020, p. 117-118).

Todo esse aparato engloba um público bastante heterogêneo de estudantes, não só pelo tipo de dificuldade (física, mental, intelectual, sensorial) mas também pelo grau com que ela se manifesta (do leve ao severo). Destaca-se que a legislação determina que todos sejam devidamente assistidos em suas necessidades e incluídos no ambiente escolar. 
Observando-se os perfis apresentados, constata-se que o estudante diabético não compõe o grupo considerado especial, logo, não está amparado pela legislação em vigor. De fato, suas dificuldades são diferentes, mas existem e deveriam ser observadas pelas instituições escolares, pois isso também é inclusão.

Atualmente, não há lei - federal, estadual ou municipal -, que obrigue as instituições escolares a ajudarem o DM1 em sua rotina de cuidados, isto é, as escolas não são obrigadas a dispor de um profissional para auxiliar o estudante nos cálculos para a correção de sua glicemia, fazer aplicação de insulina, medir a glicemia ou ajuda-lo a monitorá-la, ou que saiba como corrigir uma hipoglicemia ${ }^{3}$. Cuidados essenciais para a sobrevivência do diabético.

Na Rede Municipal de Ensino de Cuiabá, atualmente, a única assistência ofertada é o cardápio diferenciado, elaborado conforme as necessidades nutricionais específicas dos estudantes que possuem alergias alimentares, intolerância a determinados alimentos, doença celíaca ou outras patologias dessa natureza, inclusive o diabetes. Essa assistência foi estabelecida pela Lei $\mathrm{n}^{\circ}$ 12.982/2014, que alterou o Programa Nacional de Alimentação Escolar de que trata a Lei $\mathrm{n}^{\circ}$ 11.947/2009 e é de fundamental importância. Não há dúvidas de que a alimentação é a variável de maior impacto no controle da glicemia, porém, há muitos outros fatores que precisam ser constantemente observados, como os descritos há pouco, evidentemente complexos para crianças na faixa etária dos estudantes do ensino fundamental.

Diante do exposto, torna-se imprescindível que a família procure estabelecer uma relação de parceria com a escola, a fim de promover um ambiente escolar adequado para o desenvolvimento emotivo-intelectual do estudante e seguro para sua saúde física. Nesse sentido, compete à família compartilhar com a equipe escolar informações elementares a respeito do diabetes e sobre como solucionar eventuais intercorrências. Para isso, pode lançar mão de materiais educativos elaborados com a finalidade de facilitar esse diálogo e instruir as escolas com as informações básicas sobre o DM1. Como exemplos de excelentes iniciativas, pode-se mencionar o Programa Kids - Diabetes nas Escolas, disponibilizado gratuitamente pela ADJ Diabetes Brasil ${ }^{4}$ em parceria com a Federação Internacional de Diabetes; e o curso on-line gratuito promovido pelo Centro de Referência Diabetes nas Escolas da Santa Casa $\mathrm{BH}^{5}$, que pode ser realizado por profissionais da educação de qualquer região do Brasil.

É fundamental ressaltar que somente as famílias que possuem educação em diabetes serão capazes de compartilhar essas informações. Além disso, se os responsáveis pela criança não se empenharem em aprender sobre diabetes será incoerente esperar que a equipe escolar o faça.

Feitas essas necessárias considerações, agora serão apresentados os principais sentidos produzidos pelas Mães Pâncreas sobre diabetes e sobre escola.

\section{2 $\mathrm{O}$ acesso aos insumos e o conhecimento sobre diabetes}

As quatro participantes têm em comum filhos diagnosticados com diabetes tipo 1 há mais de cinco anos. As crianças uma menina e três meninos -, possuem idade entre 6 e 12 anos e são estudantes do $1^{\circ}$ ao $7^{\circ}$ ano de escolas municipais da capital mato-grossense.

Pelo tempo de diagnóstico, todas as famílias já passaram da fase inicial ou de adaptação à nova vida, o que não significa que seja fácil manejar o tratamento, até porque as doses de insulina precisam ser constantemente ajustadas devido aos

\footnotetext{
${ }^{3}$ Hipoglicemia é a concentração muito baixa de glicose no sangue (abaixo de $70 \mathrm{mg} / \mathrm{dl}$ ) e geralmente vem acompanhada de sintomas como: suor, visão embaçada, tremores, confusão mental, fraqueza, fome, taquicardia, sonolência e tontura. Em casos severos, pode gerar comprometimento cognitivo grave, por isso deve ser rapidamente corrigida com a ingesta de carboidratos de rápida absorção. (SDB, 2020; IDF, 2014).

${ }^{4}$ A Associação de Diabetes Juvenil (ADJ) é uma entidade não-governamental que atua na conscientização e educação de pessoas com diabetes e seus familiares, bem como na capacitação de profissionais de saúde e na mobilização da população em geral. Os materiais do Programa Kids podem ser acessados pelo site https://kids.idf.org.

${ }^{5}$ Informações sobre o projeto Diabetes nas escolas estão disponíveis no site http://diabetesnasescolas.com.br/.
} 
vários fatores que envolvem o crescimento de uma criança. Além disso, é fundamental destacar que o tratamento é extremamente individualizado, ou seja, a sensibilidade à ação das insulinas é diferente para cada paciente, assim como é diferente a resposta de cada organismo a determinados alimentos ou aos efeitos de uma atividade física, de infecções, da ansiedade etc. Em suma, nenhum dia é igual ao outro para quem vive com diabetes.

Em relação ao acesso aos insumos indispensáveis ${ }^{6}$ ao tratamento da criança, observam-se três cenários distintos: 1) famílias que retiram parte dos insumos pelo SUS e custeiam a outra parte (Mães Pâncreas 1 e 2); família que custeia sozinha os insumos (Mãe Pâncreas 3) e família que depende completamente do SUS (Mãe Pâncreas 4). Essa informação é interessante, pois apesar de existir uma lei que determina o fornecimento de insumos pelo $\mathrm{SUS}^{7}$, nem todas as famílias utilizam plenamente esse direito.

Ao serem questionadas em relação aos conhecimentos que possuíam sobre diabetes à época do diagnóstico, as participantes responderam:

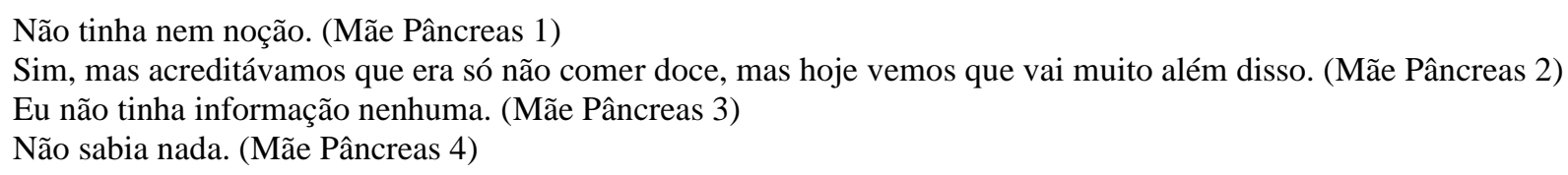

Essas declarações confirmam que as pessoas normalmente possuem pouco conhecimento sobre diabetes, conforme já afirmado neste artigo. No entanto, espera-se que após o diagnóstico o paciente ou seus familiares comecem a buscar informações sobre essa condição de saúde. Isso diz respeito à educação em diabetes, considerada pelos especialistas como uma das partes mais importantes do tratamento. Segundo Helman e Guerra (2019), refere-se ao autocuidado; ao protagonismo que o paciente - ou seu cuidador, no caso das crianças - assume em relação ao manejo do tratamento. Hoje, há muita informação de qualidade disponível gratuitamente e facilmente acessível. Todos podem aprender tanto quanto quiserem. Nesse sentido, as redes sociais podem ser valiosas fontes de informação sobre a doença, como afirmam Nass et al. (2019).

Foi possível perceber nas respostas que nem todas as mães possuem boa educação em diabetes. Ao perguntar sobre a participação do filho nas atividades escolares, uma das respostas obtidas foi a seguinte:

Ele participa de tudo. Ele pode comer de tudo, ele não pode ficar sem comer pra não ter hipoglicemia. (Mãe Pâncreas 3)

Percebe-se nessa fala que a mãe acredita que a hipoglicemia seja causada pela falta de comida e não pelo excesso de insulina, que é o correto. Dessa forma, em vez de buscar o melhor ajuste das doses a mãe acaba perpetuando o equívoco de alimentar a criança a cada 3 horas, mesmo que ela esteja sem fome.

A resposta acima contrasta com a de outra mãe que demonstra estudar sobre o DM1, como se observa:

Sim, ela participa de todas. Ela sabe da condição dela e do que deve ser feito. Sabe que precisa medir a glicose antes e depois da aula de educação física. Que precisa aplicar insulina para comer e caso estiver em hipoglicemia, que precisa de um doce. Porém, ela não sabe tomar essas decisões sozinha em relação ao que comer e a hora de comer. Se trata de um assunto muito sério e dependendo da situação ela pode chegar a ter episódio de hipoglicemia, acarretando perda da consciência, e nos casos mais graves, a óbito. (Mãe Pâncreas 2)

Essa resposta expressa que a mãe entende que o monitoramento constante da glicemia é fundamental para a tomada de decisões, e que assim é possível evitar ou diminuir as chances de hiperglicemia ou de hipoglicemia, cujas consequências

\footnotetext{
${ }^{6}$ Insulina de ação lenta (basal), insulina de ação rápida, agulhas para caneta aplicadora de insulina, fitas reagentes para teste de glicemia.

${ }^{7}$ Trata-se da Lei $n^{\circ} 11.347$, de 27/09/2006, que dispõe sobre a distribuição gratuita de medicamentos e materiais necessários à sua aplicação e à monitoração da glicemia capilar aos portadores de diabetes inscritos em programas de educação para diabéticos.
} 
podem ser bastante graves, como ela mesma relatou. Atualmente, esse monitoramento pode ser realizado de maneira prática e indolor por meio de sensores que medem continuamente os níveis de glicose, todavia, eles têm um custo bastante elevado e não são fornecidos pelo SUS. Logo, nem todas as crianças conseguem ter acesso a essa e outras tecnologias que melhoram a qualidade de vida do paciente e a adesão ao tratamento, o que reforça que o diabetes também é uma questão social ${ }^{8}$.

\subsection{A relação família-escola}

Conforme visto anteriormente, na ausência de leis que garantam assistência às necessidades da criança diabética no ambiente escolar, a parceria entre escola e família torna-se fundamental.

Dentre os filhos das participantes desta pesquisa, três foram diagnosticados antes de completarem 2 anos de idade, ou seja, já conviviam com o diabetes quando começaram a frequentar a escola. Sobre o processo de adaptação escolar, chama a atenção a declaração da Mãe Pâncreas 2:

Foi mais difícil para mim do que para ela, pois eu precisava e preciso ficar na escola para administrar insulina para o lanche e também acompanhar as glicemias, visto que só tem direito a uma TDE quem tem necessidades físicas ou psicológicas. (Mãe Pâncreas 2)

A fala dessa mãe evoca sentidos muito significativos. Primeiramente, observa-se o impacto do DM1 sobre o tempo e a rotina da Mãe pâncreas, que diante da preocupação com a saúde da filha, dispõe-se a ficar na escola durante todo o turno escolar.

Ao resgatar o perfil socioeconômico apresentado no Quadro 1, verifica-se que essa mãe, apesar de possuir curso superior completo, não está exercendo sua profissão ou qualquer outra atividade remunerada. A dificuldade de conciliar os cuidados com a criança e vida profissional força muitas mães pâncreas a renunciarem às suas carreiras, como já observado por Okido et. al (2017 citados por Brich, 2021). Somente entre as participantes deste estudo, duas declararam ser do lar e duas, autônomas, que possuem maior flexibilidade sobre seus horários de trabalho.

Outra observação se refere ao conhecimento dessa mãe acerca da legislação. Embora tenha se referido a TDE (técnico de desenvolvimento escolar) em vez de CAD (cuidador de aluno com deficiência), ela demonstra saber que as escolas municipais podem disponibilizar um profissional para auxiliarem estudantes com dificuldades no ambiente escolar. Contudo, a função desse cuidador é "auxiliar o estudante com deficiência em condições de dificuldade extrema, relacionada à autonomia na locomoção, higiene e alimentação. Inclui-se aqui o estudante com Transtorno do Espectro Autista." (Machado \& Silva, 2019, p. 175). Conforme está explícito na Política Educacional da Secretaria de Educação de Cuiabá, estudantes com diabetes não atendem aos critérios de funcionalidade que ensejam a disponibilização de um CAD.

Quanto ao apoio recebido na instituição escolar, as mães relataram experiências distintas. Para a pergunta sobre o quão preparadas as escolas estão para receberem estudantes com DM1, surgiram as seguintes respostas:

A escola não está preparada. Por eles meu filho não estudava este ano. (Mãe Pâncreas 1)

Não está, e também vejo que não tem interesse. Pois por mais que não tenha leis (ou eu as desconheça), acredito que a escola poderia ter um olhar mais carinhoso sobre os alunos com DM1. (Mãe Pâncreas 2)

Eles são bem orientados, todos eles. Eles gostam muito do meu filho. (Mãe Pâncreas 3)

Creio que sim, quando precisei eles sempre me apoiaram. A professora dele cuida muito bem dele pra mim. (Mãe Pâncreas 4)

\footnotetext{
${ }^{8} \mathrm{O}$ impacto financeiro causado pelo diabetes é sentido pelas famílias, que precisam custear os insumos que o SUS não fornece ou concede em quantidade insuficiente, e pelo governo. Como pode ser visto em SBD (2020), os custos provocados pelo diabetes podem comprometer até $20 \%$ do orçamento dos sistemas de saúde de vários países em todo o mundo.
} 
Considerando que a Política de Educação é a mesma para as quatro escolas onde os filhos das participantes estudam, depreende-se que a maneira como cada instituição acolhe o estudante depende da equipe escolar.

Como bem pontuado por Netto (2019), a educação inclusiva ainda não recebeu a merecida atenção por parte das políticas públicas brasileiras, inclusive no que se refere à formação docente, no entanto, os educadores podem tornar o processo de inclusão digno e respeitoso, demonstrando acolhimento ao estudante e sua família.

É esse acolhimento que as Mães Pâncreas relataram ansiar. As práticas discursivas evidenciaram que elas creem que o diabetes não trará prejuízos ao desenvolvimento intelectual de seus filhos, e que eles podem interagir com seus colegas e participar de todas as atividades de aprendizagem, inclusive as extraescolares. Contudo, a maior dificuldade encontrada no ambiente escolar está relacionada à falta de uma pessoa a quem possam confiar cuidados básicos da rotina diabética, como medir a glicemia, identificar sintomas de hipoglicemia e ajudar a criança a corrigi-la. A escola deveria ser parte da rede de apoio ao estudante e seus familiares. E isso não deveria depender de determinação legal para ocorrer.

\section{Considerações Finais}

Diante do exposto, ficou evidente que o diabetes tipo 1 ainda é uma condição a ser descoberta pela escola brasileira. Os dados evidenciaram a necessidade urgente do estabelecimento de políticas públicas que visem à capacitação dos profissionais da educação sobre essa condição, a fim de que possam oportunizar ao estudante diabético as mesmas condições de desenvolvimento sócio-emotivo-intelectual propostas aos demais estudantes.

Constatou-se também que a rede de apoio às Mães Pâncreas, em alguns casos, é inexistente. Mesmo aquelas que possuem educação em diabetes e condições de oferecer o tratamento adequado aos filhos necessitam de suporte, e a parceria da instituição escolar torna-se fundamental, não só pelo aspecto físico, mas também pelo emocional, igualmente importante.

Considerando que a educação é dever da família e do Estado (Brasil, 1996), a oferta de ações de educação em diabetes e saúde escolar não deveria ficar sujeita a iniciativas particulares. É um assunto de Estado e deve ser tratado como tal.

Um país que deve ambiciar maior desenvolvimento futuro não pode prescindir de todos os brasileiros para construí-lo. Sendo assim, a criança precisa ser apoiada pela escola em todos os aspectos de suas limitações e em todos os envolvimentos que precise ter.

Tendo em vista que a necessidade de receber o apoio escolar não é exclusiva dos estudantes de escolas públicas, sugere-se a realização de estudos que investiguem como se dá a inclusão de estudantes diabéticos nas escolas particulares brasileiras.

\section{Referências}

Brasil. (1990). Lei n. 8.069, de 13 de julho de 1990. Dispõe sobre o Estatuto da Criança e do Adolescente e dá outras providências. Brasília, DF. http://www.planalto.gov.br/ccivil_03/leis/18069.htm.

Brasil. (1996). Lei n. 9.394, de 20 de dezembro de 1996. Estabelece as diretrizes e bases da educação nacional. Brasília, DF. http://www.planalto.gov.br/ccivil_03/leis/L9394compilado.htm.

Brasil. (2006). Lei n. 11.347, de 27 de setembro de 2006. Dispõe sobre a distribuição gratuita de medicamentos e materiais necessários à sua aplicação e à monitoração da glicemia capilar aos portadores de diabetes inscritos em programas de educação para diabéticos. Brasília, DF. https://www.planalto.gov.br/ccivil_03/_ato2004-2006/2006/lei/111347.htm.

Brasil. (2014). Lei n. 12.982, de 28 de maio de 2014. Altera a Lei n ${ }^{\circ} 11.947$, de 16 de junho de 2009, para determinar o provimento de alimentação escolar adequada aos alunos portadores de estado ou de condição de saúde específica. http://www.planalto.gov.br/ccivil_03/_ato2011-2014/2014/lei/112982.htm

Brasil. (2015). Lei n. 13.146, de 6 de julho de 2015. Institui a Lei Brasileira de Inclusão da Pessoa com Deficiência (Estatuto da Pessoa com Deficiência). Brasília, DF. http://www.planalto.gov.br/ccivil_03/_ato2015-2018/2015/lei/113146.htm.

Brasil. (2020). PNEE: Política Nacional de Educação Especial: Equitativa, Inclusiva e com Aprendizado ao Longo da Vida. Secretaria de Modalidades Especializadas de Educação. Brasília, MEC. SEMESP. 124p. 
Research, Society and Development, v. 11, n. 2, e44411226035, 2022

(CC BY 4.0) | ISSN 2525-3409 | DOI: http://dx.doi.org/10.33448/rsd-v11i2.26035

Brich, M. (2021). "Mãe pâncreas": o cuidado de crianças e adolescentes em condição diabética. Dissertação (Mestrado) - Universidade Federal de Santa Catarina.

Guerra, E. L. A. (2014). Manual de Pesquisa Qualitativa. Grupo Ânima Educação.

Helman, B. \& Guerra, L. D. S. (2019). A relação entre a educação em saúde de pacientes com diabetes e os gastos em saúde no setor público. Journal of Management \& Primary Health Care. https://doi.org/10.14295/jmphc.v11iSup.775.

International Diabetes Federation (IDF). (2014). Pacote educativo sobre diabetes nas escolas. https://kids.idf.org/

International Diabetes Federation (IDF). (2021). IDF Diabetes Atlas. 10th ed. https://diabetesatlas.org/atlas/tenth-edition/

Lakato, E. M. \& Marconi, M. A. (2017). Fundamentos de Metodologia Científica. (8a ed.). Atlas.

Machado, E. S. \& Silva, M. S. M. (Org.). (2019). Escola Cuiabana: cultura, tempos de vida, direitos de aprendizagem e inclusão. Print Gráfica e Editora.

Maraschin, J. F., Murussi, N., Witter, V. \& Silveiro, S. P. (2009). Classificação do Diabete Melito. Arq Bras Cardiol 95(2): e40-e47. https://www.scielo.br/j/abc/a/TbH9VwBDzssTWbtwNg9LPhG/?format=pdf\&lang=pt

Nass E. M., Marcon, S.S., Teston, E. F., Reis, P., Peruzzo, H. E., Monteschio, L.V. et al. (2019). Perspectiva de jovens com diabetes sobre intervenção educativa na rede social Facebook@. Acta Paul Enferm, 32 (4), 390-397. http://dx.doi.org/10.1590/1982-0194201900054.

Neto, C. (2019). O que fazer para melhorar a inclusão nas escolas? Revista Nova Escola. Recuperado de: https://gestaoescolar.org.br/conteudo/2293/o-quefazer-para-melhorar-a-inclusao-nas-escolas

Sociedade Brasileira de Diabetes (SBD). (2020). Diretrizes da Sociedade Brasileira de Diabetes 2019-2020. Clannad Editora Científica.

Spink, M. J. (2010). Linguagem e produção de sentidos no cotidiano. Centro Edelstein de Pesquisas Sociais, 2010. 72 p.

Spink, M. J. (Org.). (2013). Práticas discursivas e produção de sentidos no cotidiano. Edição virtual. Centro Edelstein de Pesquisas Sociais.

Vinha, T. (2016). O papel da escola e da família na formação das crianças. Revista Nova Escola, 289. https://novaescola.org.br/conteudo/8553/o-papel-daescola-e-da-familia-na-formacao-das-criancas. 